\title{
27. X-RAY MINERALOGY FROM HOLES 399, 400, 400A, 401, 402 AND 402A OF BAY OF BISCAY
}

\author{
G. Cassat, Société Nationale des Pétroles d'Aquitaine, Pau, France
}

\section{INTRODUCTION}

Routine X-ray mineralogy analysis of 279 samples from Holes 399, 400, 400A, 401, 402, and 402A in the Bay of Biscay have been performed in order to ascertain shipboard lithological determinations.

\section{Methodology}

Quartz, calcite, and dolomite have been analyzed automatically in percentages versus total rock (see Figures 1,2 , and 3 ). (Figure 1 is in back pocket, this volume.)

The presence of siderite, $\mathrm{Mg}$-calcite, and aragonite has been verified (see Tables 1 and 2). In these tables of X-ray mineralogy results, the aragonite fraction has been quantitatively dosed as follows: 1 signifies zero to 10 per cent of the total rock; $2=10$ to 50 per cent; $3=50$ to 80 per cent; and $4=$ greater than 80 per cent. The occurrence of these carbonates and the poor crystallinity of calcite (particularly in the Hole 402A samples of Albian-Aptian sediment) probably explains the large differences between our data and the carbonate content analysis of the shipboard party.

We have determined the clay mineral composition (kaolinite, illite, illite-smectite, smectite, and chlorite) of the carbonate-free, fine-grained fraction $(<5 \mu \mathrm{m})$. In addition, we frequently have found zeolites from the late Aptian up to the Eocene, and opal-CT from the late Aptian up to the late Albian. These minerals are analyzed on the carbonate-free fine-fraction, together with the clay minerals. The major part of the zeolites seems to be clinoptilolite, but some diagrams are different, e.g., Sample $402 \mathrm{~A}-23-3,104 \mathrm{~cm}$. We always measured the highest peak, which is generally located at $2 \Theta=10^{\circ}$.

We did not definitely prove the presence of fibrous clays, which are mentioned by Chamley (this volume); this can be explained by their low content $(<5 \%)$. Nevertheless, we have mentioned the samples whose diagrams remained uncertain. These samples are indicated by the notation " 1 " in the columns "attapulgite" and "sepiolite." (See Tables 1 and 2.)

Finally, we have recorded the presence of fine-grained quartz and feldspars, with the same notation as for the carbonates in the total rock.

\section{SUMMARY OF RESULTS}

\section{Holes 399, 400, and 400A}

The following three major sedimentary sequences can be defined for these holes: Lower Cretaceous, Upper Cretaceous and lower Tertiary, and upper Tertiary and Quaternary.

\section{Lower Cretaceous}

These sediments are generally beige to dark gray, slightly silty, calcareous clays or marls with ligneous remains. Smectite largely prevails, with illite and illite-smectite. Zeolites and opal-CT are intermittent and locally abundant (see Plate 1, Figures 1 and 2).

The samples are alternately more silty and less carbonated, especially during the lower to middle Albian. In contrast, the upper Albian is generally more carbonated and contains a larger amount of opal-CT.

\section{Upper Cretaceous-Lower Tertiary}

These deposits are generally ochre to beige, silty calcareous clays. These samples are more silty and calcareous than those of the Lower Cretaceous. Smectite is still largely dominant, with some illite and kaolinite, and a low amount of chlorite. Illite-smectite has disappeared. The zeolite content is still high during the Upper Cretaceous, and it becomes intermittent above this horizon. Opal-CT has completely disappeared.

\section{Upper Tertiary and Quaternary}

This sequence is composed of gray-white to white, slightly silty marls up to the middle of the upper Pliocene: The sediments are much more silty and locally much less carbonated above this horizon. A slight occurrence of siderite can be noted.

Smectite decreases regularly, with an increase of illite during the Oligocene and an increase of illite-smectite and chlorite during the Pliocene.

Samples 400A-12-2, $106 \mathrm{~cm}$ and 400A-44-1, $107 \mathrm{~cm}$ are particularly rich in fine-grained silica. For the former, this could be a silt ( $38 \%$ of quartz); for the latter, a beginning of silicification.

\section{Site 401 (Plates 1 and 2)}

These samples are much more carbonated and less silty in Site 401 .

\section{Lower Cenomanian}

Only one sample, with ochre argillaceous micrite, is identified as being lower Cenomanian. Smectite is largely predominant with illite-smectite and minor chlorite and kaolinite.

\section{Maestrichtian-Campanian}

This sediment is characterized by white argillaceous micrite. Smectite is dominant with illite, and there are occurrences of zeolite. 


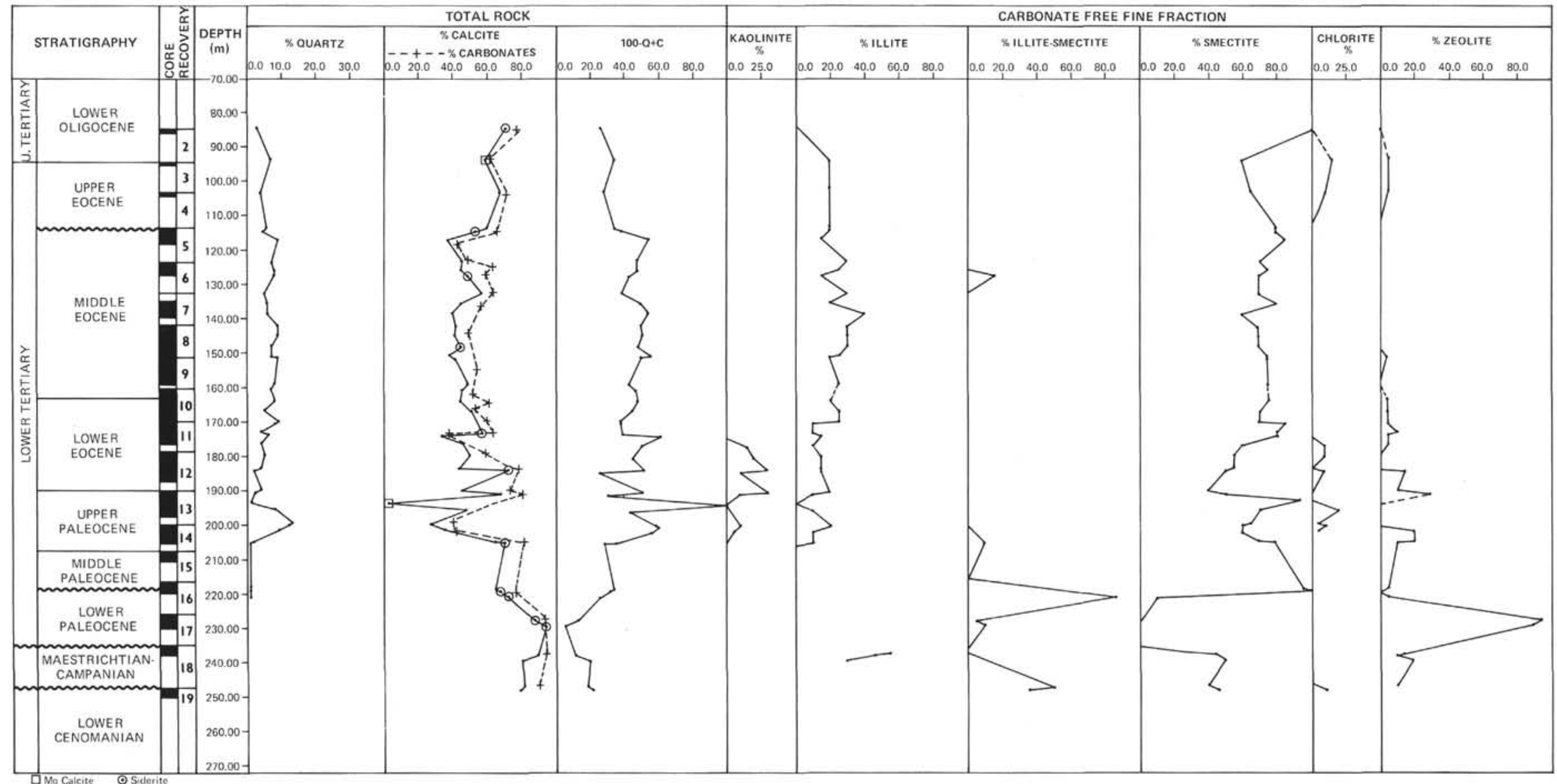

Figure 2. X-ray mineralogy for IPOD Leg 48, Site 401. 


\section{Lower Tertiary}

A regular decrease in the calcite content can be noted up to the upper Eocene, with an increase of the silt fraction.

Smectite is definitely dominant, with a slight increase in illite, and occurrences of kaolinite and chlorite in the upper Paleocene and lower Eocene.

The zeolites are very common up to the lower part of the middle Eocene.

An increase in carbonate content is noticeable in the upper Eocene and lower Oligocene. Smectite is the only clay mineral in the lower Oligocene.

\section{Quaternary}

The samples are composed of gray, gray-beige, and beige to dark gray silty calcareous marls, with illite dominant, and with illite-smectite, kaolinite, and chlorite.

\section{Holes 402 and 402A (Plate 2)}

Three intervals can be distinguished (with clearcut breaks of sedimentation) between Lower Cretaceous, middle to upper Eocene, and Quaternary.

\section{Lower Cretaceous}

This interval is composed of gray or beige to dark gray, silty shales, with abundant ligneous debris.
The presence of white micrite (Sample 402-6-1, $43 \mathrm{~cm}$ ) and siderite concretion (Samples 402-19-4, $106 \mathrm{~cm}$ and $402 \mathrm{~A}-21-4,111 \mathrm{~cm}$ ) can be noted. Siderite is locally abundant in the Gargasian and the Bedoulian. Aragonite principally occurs from the base of the upper Gargasian up to the base of the upper Albian.

Some horizons rich in Mg-calcite (Samples 402A-31-3, $68 \mathrm{~cm}$ and $402 \mathrm{~A}-34-1,82 \mathrm{~cm}$ ) have been observed. These concentrations are probably linked with the presence of numerous shell debris.

Smectite is largely predominant, with some kaolinite and illite; there are slightly chlorite occurrences at the base of the Gargasian. From the uppermost Aptian and above, the zeolite and opal-CT contents increase.

\section{Middle to Upper Eocene}

Gray-beige silty marls, with largely predominant silica and badly crystallized illite, occurred at the base of this unit, up to Sample 402A-3-2, $68 \mathrm{~cm}$; no illite was found until Sample $402-5-3,63 \mathrm{~cm}$. There is no kaolinite, chlorite, nor zeolite.

\section{Quaternary}

The sediments of this age have the same appearance as their equivalents in the other sites. 


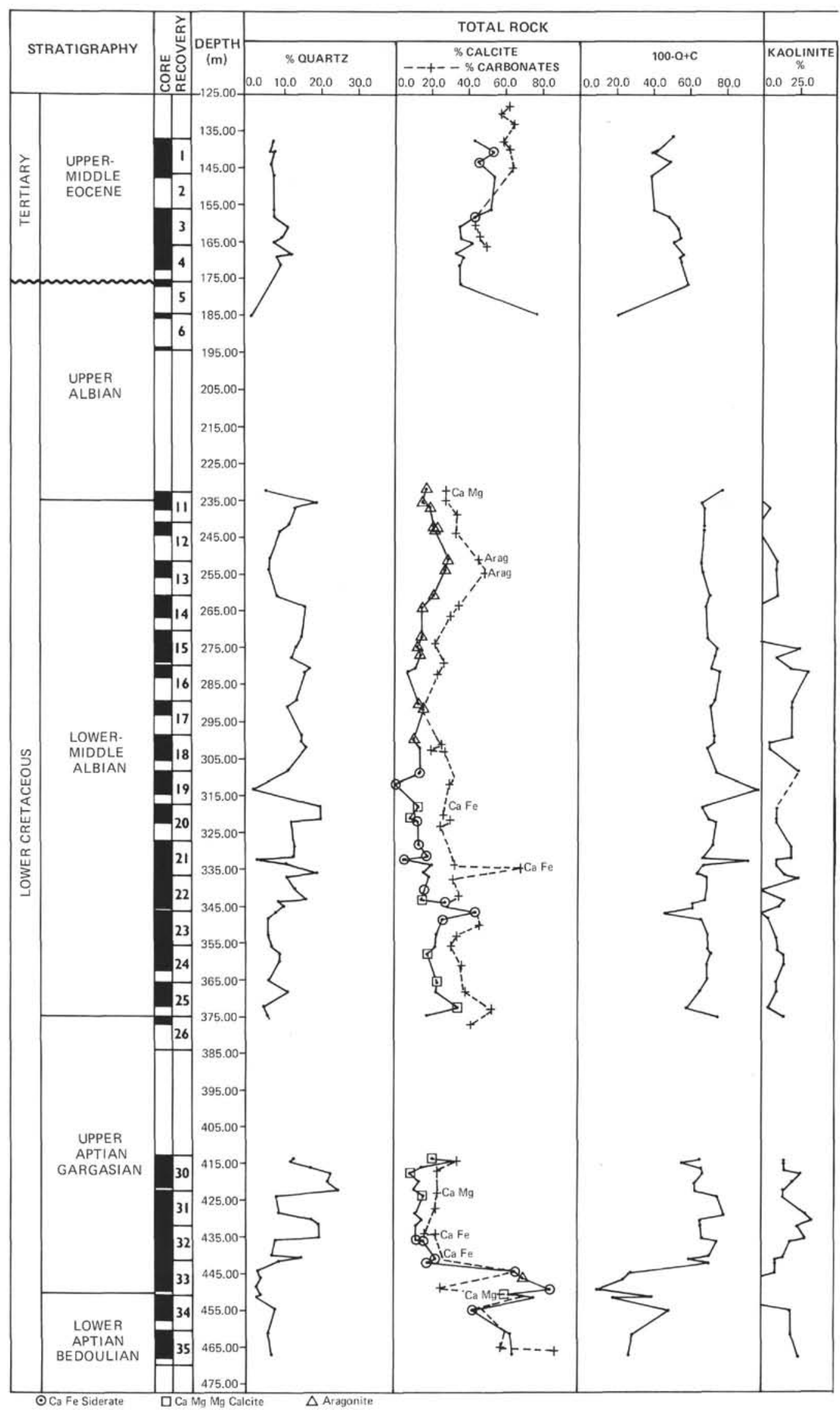

Figure 3. X-ray mineralogy for IPOD Leg 48, Hole $402 A$. 


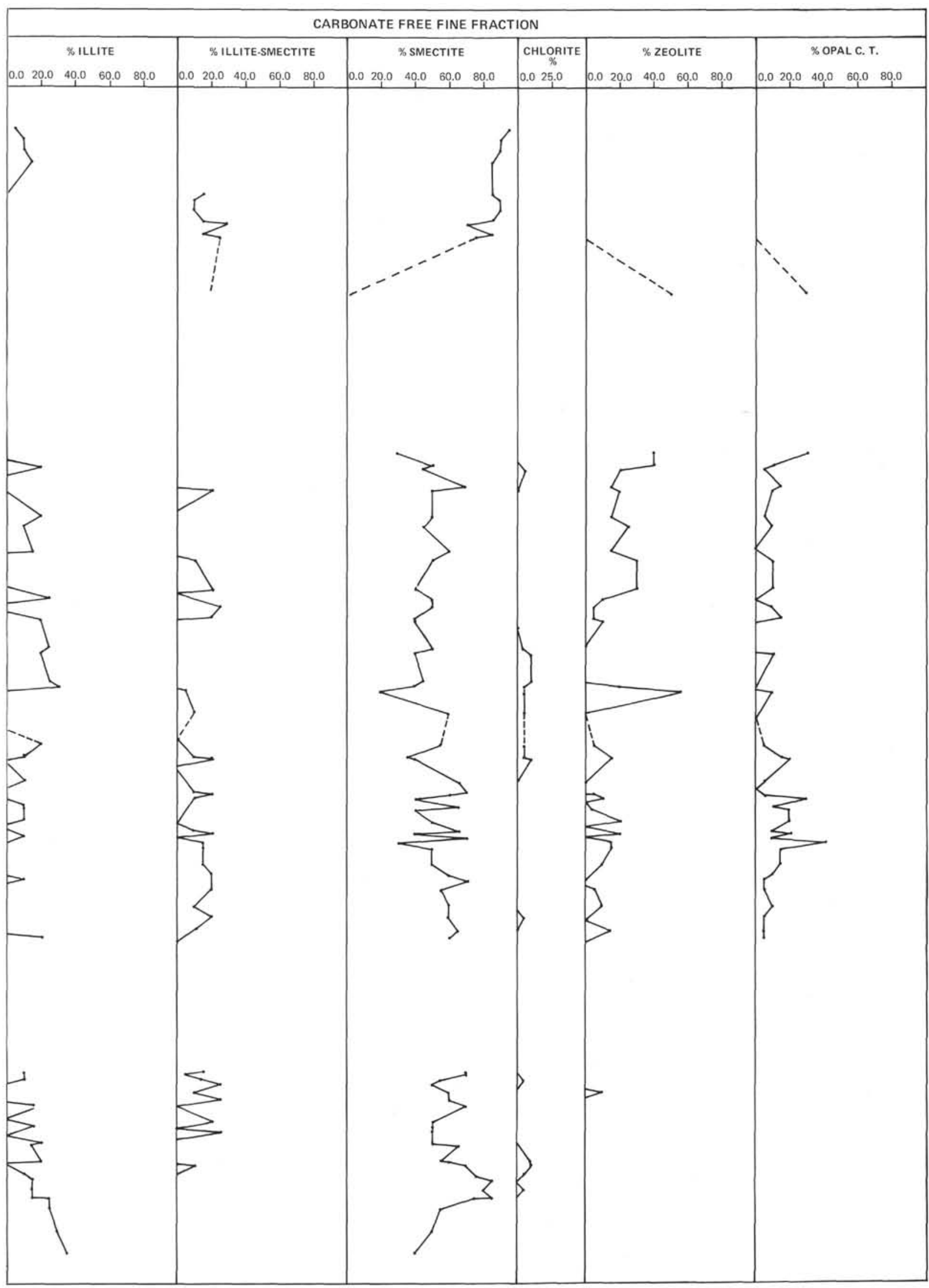

Figure 3. (Continued). 


\begin{tabular}{|c|c|c|c|c|c|c|c|c|c|c|c|c|c|c|c|c|c|c|c|c|c|c|c|c|c|c|c|c|c|c|}
\hline \multirow[b]{4}{*}{ Stratigaphy } & \\
\hline & \multicolumn{11}{|c|}{ Holes 399,400 , and $400 \mathrm{~A}$} & \multicolumn{8}{|c|}{ Site 401} & \multicolumn{11}{|c|}{ Site 402} \\
\hline & \multirow[b]{2}{*}{ Sample } & \multicolumn{3}{|c|}{\begin{tabular}{|l|} 
Total Rock \\
\end{tabular}} & \multicolumn{7}{|c|}{ Carbonate-Free Fraction } & \multirow[b]{2}{*}{ Sample } & \multicolumn{2}{|c|}{ Total Rock } & \multicolumn{5}{|c|}{ Carbonate-Free Fraction } & \multirow[b]{2}{*}{ Sample } & \multicolumn{3}{|c|}{ Total Rock } & \multicolumn{7}{|c|}{ Carbonate-Free Fraction } \\
\hline & & $\begin{array}{ll}8 \\
8\end{array}$ & & 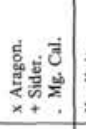 & 善部 & 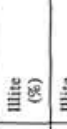 & 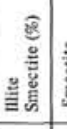 & 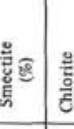 & 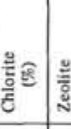 & 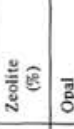 & 8 & & 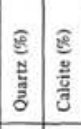 & 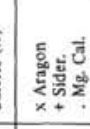 & 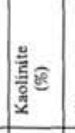 & 旁里 & 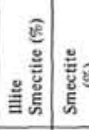 & 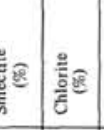 & 彦 & & & 高 & 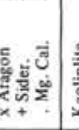 & 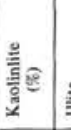 & & 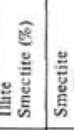 & 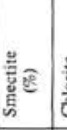 & 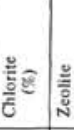 & 善 & 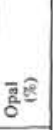 \\
\hline 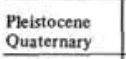 & & 143 & 31 & +2 & 15 & 35 & 17 & \begin{tabular}{l|l}
13 & 2 \\
\end{tabular} & 20 & & & & \begin{tabular}{l|l}
${ }_{22}^{23}$ & 20 \\
\end{tabular} & $\begin{array}{l}0+ \\
\end{array}$ & 10 & 40 & 25 & 25 & & & 28 & & dol.1. & 16 & 39 & \begin{tabular}{l|l}
20 & 1
\end{tabular} & 10 & 15 & & \\
\hline $\begin{array}{l}\text { Upper } \\
\text { Pliocene }\end{array}$ & & \begin{tabular}{c|c}
14 & 3 \\
7 & 5 \\
7
\end{tabular} & $\begin{array}{c}34 \\
52\end{array}$ & +3 & $\begin{array}{l}16 \\
18 \\
\end{array}$ & $\begin{array}{l}32 \\
33\end{array}$ & $\begin{array}{l}28 \\
27\end{array}$ & \begin{tabular}{l|l}
6 & 1 \\
6 & 1 \\
\end{tabular} & $\begin{array}{l}18 \\
16\end{array}$ & & & & & & & & & & & & & & & & & & & & & \\
\hline 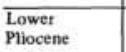 & & \begin{tabular}{|l|l}
8 & 5
\end{tabular} & 57 | & & 14 & 34 & 7 & \begin{tabular}{l|l}
28 & 1 \\
\end{tabular} & 17 & & & & & & & & & & & & & & & & & & & & & \\
\hline $\begin{array}{l}\substack{\text { Upper } \\
\text { Mioerne }} \\
\text { D. }\end{array}$ & & 75 & 55 & & & & & & & & & & & & & & & & & & & & & & & & & & & \\
\hline $\begin{array}{l}\text { Middle } \\
\text { Miocene }\end{array}$ & $\begin{array}{l}25.6 .6115 \\
36\end{array}$ & 36 & 66 & +3 & 16 & 57 & & 41 & 6 & & & & & & & & & & & & & & & & & & & & & \\
\hline $\begin{array}{l}\text { Lower } \\
\text { Miocene }\end{array}$ & $\begin{array}{l}37 \\
41\end{array}$ & \begin{tabular}{l|l}
4 & 5
\end{tabular} & 52 & +1 & 14 & 31 & & so & $\mathrm{s}$ & & & & & & & & & & & & & & & & & & & & & \\
\hline $\begin{array}{l}\begin{array}{l}\text { Upper-Middle } \\
\text { Ollaocene }\end{array} \\
\end{array}$ & $\begin{array}{l}43 \\
45-4,74\end{array}$ & ${ }_{4}$ s & 53 & +1 & 21 & 21 & & 54 & 4 & & & & & & & & & & & & & & & & & & & & & \\
\hline $\begin{array}{l}\text { Lower } \\
\text { Eocene }\end{array}$ & $\begin{array}{l}45, \mathrm{cc} \\
46\end{array}$ & 75 & 53 & & 16 & 18 & & 65 & 1 & & & $\underbrace{}_{2-1,7}$ & \begin{tabular}{l|l|l}
3 & 71
\end{tabular} & 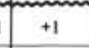 & & & & 99 & & & & & & & & & & & & \\
\hline $\begin{array}{l}\text { Upper } \\
\text { Eocene }\end{array}$ & & 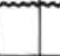 & & & & & & & & & & $\begin{array}{l}3 \cdot 1,15 \\
4, \mathrm{cC} \\
\end{array}$ & \begin{tabular}{|l|l|}
5 & 63 \\
\end{tabular} & +1 & & 20 & & \begin{tabular}{l|l}
62 & 13 \\
\end{tabular} & 5 & $5-1,65$ & 7 & 52 & & & 9 & \begin{tabular}{l|l}
2 & $8:$ \\
\end{tabular} & 89 & & & \\
\hline 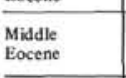 & $\begin{array}{c}47 \\
52: 1,64\end{array}$ & \begin{tabular}{r|r}
4 & \\
10 & 2
\end{tabular} & \begin{tabular}{r|r}
9 \\
22
\end{tabular} & & $\begin{array}{r}12 \\
6 \\
\end{array}$ & $\begin{array}{l}{ }_{33}^{25} \\
\end{array}$ & & $\begin{array}{c}55 \\
57\end{array}$ & $\begin{array}{l}5 \\
3\end{array}$ & & & \begin{tabular}{c|}
5 \\
$11-1,42$ \\
\end{tabular} & 84 & & & 24 & & 75 & 2 & $402 \wedge 4$ & 9 & 38 & & & & $\begin{array}{lll}17 & 8 \\
\end{array}$ & 83 & & & \\
\hline $\begin{array}{l}\text { Lower } \\
\text { Eocene } \\
\end{array}$ & $\begin{array}{c}3.57 \\
56 \\
\end{array}$ & 61 & ${ }_{18}:$ & .02 & \begin{tabular}{|l|}
8 \\
24 \\
\end{tabular} & $\begin{array}{l}15 \\
17 \\
\end{array}$ & 12 & $\begin{array}{l}50 \\
42 \\
\end{array}$ & 3 & $\begin{array}{l}9 \\
8 \\
\end{array}$ & & $\begin{array}{l}3.9 \\
12 \\
\end{array}$ & \begin{tabular}{|l|l|}
5 & 45 \\
4 & 53 \\
\end{tabular} & & 25 & 14 & & \begin{tabular}{l|l|}
55 & 8 \\
\end{tabular} & ? & & & & & & & & & & & \\
\hline $\begin{array}{l}\text { Upper } \\
\text { Paleocene }\end{array}$ & $\begin{array}{l}57 \\
58\end{array}$ & 15 & 20 & & 11 & 29 & & $49 \quad 1$ & 11 & & & $\begin{array}{l}13 \\
14 \\
\end{array}$ & \begin{tabular}{l|l}
7 & 37 \\
1 & 66 \\
\end{tabular} & & 7 & $\begin{array}{c}13 \\
5\end{array}$ & \begin{tabular}{l|l}
5 & 73 \\
5 & 75 \\
\end{tabular} & 6 & ${ }_{15}^{8}$ & & & & & & & & & & & \\
\hline $\begin{array}{l}\text { Middle } \\
\text { Paleocene }\end{array}$ & $\begin{array}{l}59 \\
59\end{array}$ & $\begin{array}{rl}13 & 3 \\
2 & 6 \\
\end{array}$ & $\begin{array}{l}34 \\
61\end{array}$ & & 8 & 18 & - & 57 & \begin{tabular}{l|l}
7 & 1
\end{tabular} & $\stackrel{10}{-}$ & & \begin{tabular}{|c|}
16 \\
$16-1,146$ \\
\end{tabular} & \begin{tabular}{l|l}
167 \\
\end{tabular} & .01 & & & & 97 & 2 & & & & & & & & & & & \\
\hline $\begin{array}{l}\text { Lower } \\
\text { Palkocenene }\end{array}$ & & & & & & & & & & & & & \begin{tabular}{r|l}
1 & 74 \\
0 & 90 \\
\end{tabular} & +5 & & & \begin{tabular}{c|c}
85 & 10 \\
7 &
\end{tabular} & m & $\begin{array}{c}5 \\
93 \\
93\end{array}$ & & & & & & & & & & & \\
\hline $\begin{array}{l}\text { Masstrichtian } \\
\text { Campanian }\end{array}$ & $\begin{array}{l}60 \\
61\end{array}$ & 3 & 65 & & 5 & 10 & & 50 & & 30 & & 18 & \begin{tabular}{l|l}
0 & 89 \\
0 & 83 \\
\end{tabular} & & & $\begin{array}{l}55 \\
24 \\
24\end{array}$ & \begin{tabular}{c|c}
30 \\
16 & 45 \\
\end{tabular} & $\begin{array}{l}30 \\
43 \\
3\end{array}$ & $\begin{array}{l}15 \\
13 \\
\end{array}$ & & & & & & & & & & & \\
\hline $\begin{array}{l}\text { Lower } \\
\text { Cenoman. }\end{array}$ & & & & & & & & & & & & & (79 & & 10 & & & 45,10 & & & & & & & & & & & & \\
\hline $\begin{array}{l}\text { Upper } \\
\text { Albian }\end{array}$ & 62 & 3 & 43 & & & 10 & 6 & 38 & & \begin{tabular}{l|l}
20 & 2
\end{tabular} & 26 & & & & - & & & & no smple & $6-1,43 \mathrm{~cm}$ & ? & 39 & $\overline{\mathbf{x}} 1 \overline{0}$ & - & . & 20. & - & -5 & $\underline{s}_{-}$ & 30 \\
\hline & 643,28 & & & & & & & & & & & & & & & & & & & & 12 & 15 X & & & & & 40 & & 40 & 20 \\
\hline 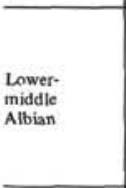 & 3,14 & 91 & 10 & +2 & & 21 & \begin{tabular}{l|l}
4 & 3
\end{tabular} & 37 & & 33 s & 5 & & & & & & & & & $\begin{array}{l}4.57 \\
14-1,62 \mathrm{~cm} \\
3.42 \\
22 \cdot 5,75 \mathrm{~cm} \\
6-14 \\
14 \\
\end{array}$ & \begin{tabular}{|l|l}
9 & 2 \\
14 & 1 \\
8 & 2
\end{tabular} & 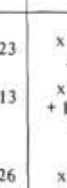 & $\begin{array}{c}\times 10 \\
.05 \\
\times 9 \\
+10 \\
.01 \\
\times 9 \\
\times 9 \\
\end{array}$ & $\begin{array}{l}6 \\
14 \\
10\end{array}$ & \begin{tabular}{c|c}
11 & \\
9 & \\
2 & 1
\end{tabular} & \begin{tabular}{c|c}
3 & 5 \\
7 & 5 \\
& \\
13 & 4
\end{tabular} & $\begin{array}{l}55 \\
50 \\
43\end{array}$ & \begin{tabular}{l|l}
18 & 1
\end{tabular} & $\begin{array}{c}18 \\
9 \\
8\end{array}$ & 8 \\
\hline & 68 & & & & & & & & & & & & & & & & & & no simple & $\begin{array}{r}24 \\
25 \\
-26 \\
-2 .-\end{array}$ & \begin{tabular}{|c|c|c|}
8 & 2 \\
7 & 2 \\
-2
\end{tabular} & & $\begin{array}{r}\times 9 \\
\times 12 \\
-.02 \\
\end{array}$ & 10 & 5 & 10 & $\begin{array}{r}43 \\
62 \\
-\end{array}$ & & 5 & 13 \\
\hline $\begin{array}{l}\text { Aower } \\
\text { Aptian }\end{array}$ & & & 25 & .01 & & 26 & & 63 & & $\begin{array}{ll}7 & 3\end{array}$ & 3 & & & & & & & & onsimen & $\begin{array}{c}30 \\
33-1,78 \mathrm{~cm} \\
3-38 \\
34 \\
34-1,82 \mathrm{~cm}\end{array}$ & \begin{tabular}{|l|l}
15 & 1 \\
3 & 7 \\
4 & 6 \\
7 & 5
\end{tabular} & \begin{tabular}{l|l}
75 & $x$ \\
65 & $\vdots$ \\
57 & $\vdots$ \\
6
\end{tabular} & $\begin{array}{l}\times 2 \\
+3 \\
.03 \\
+1 \\
.03 \\
.+5 \\
.05\end{array}$ & $\begin{array}{l}20 \\
3 \\
20\end{array}$ & \begin{tabular}{l|l}
8 & 1 \\
16 & \\
20 & \\
30 &
\end{tabular} & $10 \mid \begin{array}{l}6 \\
8 \\
8\end{array}$ & $\begin{array}{l}60 \\
80 \\
80 \\
50\end{array}$ & $\begin{array}{l}2 \\
3\end{array}$ & 1 & \\
\hline
\end{tabular}


TABLE 2

X-Ray Mineralogy Comprehensive Results, IPOD Leg 48A

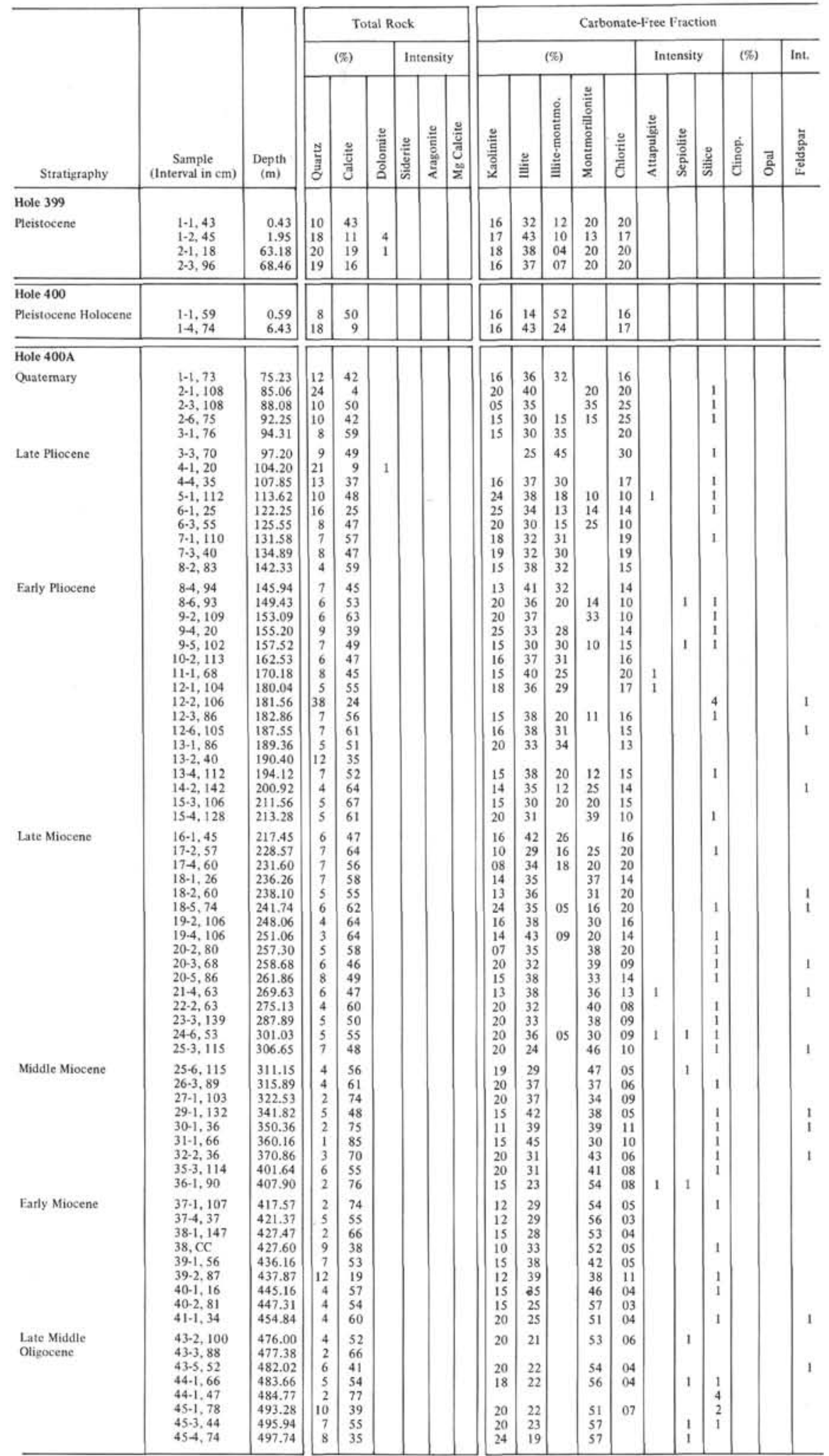


TABLE 2 - Continued

\begin{tabular}{|c|c|c|c|c|c|c|c|c|c|c|c|c|c|c|c|c|c|c|}
\hline \multirow[b]{3}{*}{ Stratigraphy } & \multirow[b]{3}{*}{$\begin{array}{c}\text { Saruple } \\
\text { (Interval in } \mathrm{cm} \text { ) }\end{array}$} & \multirow[b]{3}{*}{$\begin{array}{c}\text { Depth } \\
(\mathrm{m})\end{array}$} & \multicolumn{5}{|c|}{ Total Rock } & \multicolumn{11}{|c|}{ Carbonate-Free Fraction } \\
\hline & & & \multicolumn{3}{|c|}{ (\%) } & \multicolumn{2}{|c|}{ Intensity } & \multicolumn{5}{|c|}{ (\%) } & \multicolumn{3}{|c|}{ Intensity } & \multicolumn{2}{|c|}{ (\%) } & \\
\hline & & & 产 & $\frac{\tilde{g}}{\tilde{J}}$ & $\frac{1}{a}$ & 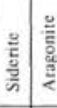 & 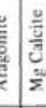 & $\frac{g}{g}$ & 㪯 & 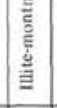 & 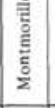 & 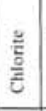 & 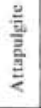 & 产 & 总 & है & & \\
\hline Eatly Oligocene & $\begin{array}{l}45, C C \\
46-4.80 \\
46-5.80 \\
46-6.8\end{array}$ & $\begin{array}{c}499.50 \\
507.30 \\
508.80 \\
509.58\end{array}$ & $\begin{array}{r}10 \\
5 \\
5 \\
6\end{array}$ & \begin{tabular}{|l|}
36 \\
58 \\
57 \\
62
\end{tabular} & & & & $\begin{array}{l}24 \\
15 \\
15 \\
12\end{array}$ & \begin{tabular}{|l|l}
4 & 24 \\
5 & 18 \\
5 & 19 \\
2 & 14
\end{tabular} & & $\begin{array}{l}58 \\
67 \\
66 \\
69\end{array}$ & 05 & 1 & & & & & \\
\hline Middle Eocene & $\begin{array}{l}47 \cdot 1,96 \\
47-6,75 \\
47-7.52 \\
48-2,114 \\
49 \cdot 1,144 \\
49-2,94 \\
49-3,33 \\
49, C C \\
50, C C \\
51-1,60 \\
51-3,36 \\
51-5,114 \\
51-7,38 \\
52-1,64\end{array}$ & $\begin{array}{l}512.46 \\
519.75 \\
521.02 \\
523.64 \\
531.94 \\
532.94 \\
533.83 \\
536.00 \\
540.00 \\
549.60 \\
552.36 \\
556.14 \\
556.88 \\
559.64\end{array}$ & $\begin{aligned} 13 \\
14 \\
10 \\
15 \\
13 \\
11 \\
13 \\
13 \\
10 \\
9 \\
11 \\
9 \\
13 \\
13 \\
8\end{aligned}$ & $\begin{array}{r}7 \\
6 \\
14 \\
2 \\
2 \\
17 \\
20 \\
8 \\
1 \\
17 \\
26 \\
20 \\
29 \\
14 \\
28\end{array}$ & & & & $\begin{array}{l}08 \\
10 \\
16 \\
12 \\
15 \\
10 \\
10 \\
12 \\
10 \\
07 \\
\\
10 \\
10\end{array}$ & 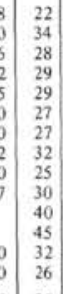 & & $\begin{array}{l}66 \\
51 \\
56 \\
53 \\
51 \\
57 \\
58 \\
50 \\
59 \\
59 \\
60 \\
55 \\
54 \\
59\end{array}$ & $\begin{array}{l}04 \\
05 \\
06 \\
05 \\
06 \\
05 \\
06 \\
06 \\
04 \\
\\
04 \\
05\end{array}$ & 1 & & $\begin{array}{l}1 \\
1 \\
1 \\
1\end{array}$ & & & $\begin{array}{l}1 \\
1 \\
1 \\
1\end{array}$ \\
\hline Early Eocene & $\begin{array}{l}52-3,37 \\
52-5,42 \\
53-1,84 \\
53-2,2,2 \\
54-1,132 \\
54-2,111 \\
55-1,104 \\
55-2,46 \\
56-2,20\end{array}$ & $\begin{array}{l}562.37 \\
565.42 \\
569.34 \\
571.52 \\
579.32 \\
580.61 \\
588.64 \\
589.46 \\
598.70\end{array}$ & \begin{tabular}{|l|}
6 \\
6 \\
4 \\
6 \\
8 \\
6 \\
6 \\
5 \\
5
\end{tabular} & $\begin{array}{r}24 \\
15 \\
15 \\
8 \\
7 \\
16 \\
14 \\
32 \\
33\end{array}$ & & $\begin{array}{l}1 \\
1\end{array}$ & & $\begin{array}{l}10 \\
14 \\
08 \\
31 \\
25 \\
14 \\
25\end{array}$ & \begin{tabular}{l|l}
0 & 30 \\
4 & 31 \\
8 & \\
1 & 25 \\
5 & 20 \\
4 & 23
\end{tabular} & 21 & $\begin{array}{l}40 \\
46 \\
49 \\
65 \\
44 \\
41 \\
38 \\
44\end{array}$ & $\begin{array}{l}05 \\
09 \\
07 \\
\\
13\end{array}$ & $\begin{array}{l}1 \\
1 \\
1\end{array}$ & $\begin{array}{l}1 \\
1 \\
1 \\
1\end{array}$ & $\begin{array}{l}1 \\
1\end{array}$ & $\begin{array}{l}15 \\
15 \\
05 \\
14 \\
12 \\
06\end{array}$ & & i \\
\hline Late Paleocene & $\begin{array}{l}57, \mathrm{CC} \\
58, \mathrm{cc}\end{array}$ & $\begin{array}{l}609.50 \\
614.00\end{array}$ & $\begin{array}{l}15 \\
14\end{array}$ & $\begin{array}{l}21 \\
22\end{array}$ & & & & 11 & 29 & & 49 & 11 & 1 & & & & & \\
\hline $\begin{array}{l}\text { Middle Paleocene } \\
-\end{array}$ & $\begin{array}{l}59-1,4 \\
59 \cdot 1,23\end{array}$ & $\begin{array}{l}625.54 \\
625.73 \\
\end{array}$ & $\begin{array}{r}13 \\
2\end{array}$ & $\begin{array}{r}34 \\
61 \\
61\end{array}$ & & & & 08 & \begin{tabular}{l|l}
3 & 18 \\
\end{tabular} & & 57 & 07 & & & 1 & 10 & & 1 \\
\hline $\begin{array}{l}\text { Maest-Campanian } \\
-\end{array}$ & $\begin{array}{l}60-7.8 \\
61 . \mathrm{CC}\end{array}$ & $\begin{array}{l}644.08 \\
654.00 \\
6\end{array}$ & $\begin{array}{l}2 \\
4 \\
\end{array}$ & $\begin{array}{l}79 \\
53\end{array}$ & & & & & & & & & & & & & & \\
\hline Late Albian & $\begin{array}{l}62-1,90 \\
62-3,10 \\
62-5,34 \\
63-1,68 \\
63-3,53 \\
63, \mathrm{CC} \\
64-1,76 \\
64-3,28 \\
64-3,3114 \\
64-5,75 \\
65-1.92\end{array}$ & $\begin{array}{l}654.90 \\
6557.10 \\
660.34 \\
6654.18 \\
667.03 \\
6699.50 \\
673.76 \\
676.28 \\
6777.14 \\
697.75 \\
683.42\end{array}$ & $\begin{array}{r}3 \\
4 \\
2 \\
3 \\
3 \\
3 \\
3 \\
4 \\
10 \\
7 \\
11\end{array}$ & $\begin{array}{l}36 \\
48 \\
51 \\
50 \\
44 \\
44 \\
41 \\
31 \\
23 \\
23\end{array}$ & & $\begin{array}{l}1 \\
1 \\
1\end{array}$ & 1 & & $\begin{array}{l}25 \\
20 \\
18\end{array}$ & $\begin{array}{l}13 \\
23\end{array}$ & $\begin{array}{l}20 \\
33 \\
35 \\
27 \\
33 \\
75 \\
70 \\
41\end{array}$ & & 1 & 1 & & \begin{tabular}{l|}
15 \\
20 \\
22 \\
13 \\
40 \\
11 \\
10 \\
10 \\
41
\end{tabular} & \begin{tabular}{l|}
65 \\
15 \\
15 \\
60
\end{tabular} & \\
\hline Early Middle Albian & $\begin{array}{l}65-1,92 \\
65-2,48 \\
66-1,100 \\
66-2,84 \\
66-4,12 \\
67-1,75\end{array}$ & $\begin{array}{l}683.42 \\
6884.48 \\
693.00 \\
6994.34 \\
696.62 \\
702.25 \\
\end{array}$ & $\begin{array}{r}11 \\
6 \\
5 \\
8 \\
12 \\
10\end{array}$ & $\begin{array}{r}1 \\
25 \\
3 \\
17 \\
3 \\
5\end{array}$ & & $\begin{array}{l}1 \\
1\end{array}$ & & $\begin{array}{l}1 \\
1 \\
1\end{array}$ & $\begin{array}{l}18 \\
20 \\
40 \\
25 \\
22\end{array}$ & 30 & $\begin{array}{l}41 \\
35 \\
31 \\
23 \\
22\end{array}$ & & & $\begin{array}{l}1 \\
1 \\
1\end{array}$ & $\begin{array}{l}1 \\
1 \\
1 \\
1\end{array}$ & \begin{tabular}{l|}
41 \\
45 \\
60 \\
52 \\
56
\end{tabular} & 39 & \\
\hline $\begin{array}{l}\text { Late Aptian } \\
\text { Gargasian }\end{array}$ & $\begin{array}{l}68-1,81 \\
68-2,71 \\
68-2,106 \\
69, \mathrm{CC} \\
70-2,92 \\
70, \mathrm{CC} \\
71-2,38 \\
71, \mathrm{CC} \\
72-2,47 \\
72-4,27 \\
74, \mathrm{CC} \\
\end{array}$ & \begin{tabular}{|}
711.81 \\
714.21 \\
714.46 \\
720.50 \\
730.00 \\
732.42 \\
739.50 \\
7391.38 \\
750.97 \\
753.77 \\
768.00 \\
\end{tabular} & \begin{tabular}{|c|}
6 \\
8 \\
6 \\
6 \\
7 \\
7 \\
6 \\
6 \\
8 \\
7 \\
6 \\
6 \\
10
\end{tabular} & \begin{tabular}{|r|}
37 \\
13 \\
35 \\
39 \\
39 \\
24 \\
28 \\
23 \\
26 \\
8 \\
\end{tabular} & & & & 1 & $\begin{array}{l}15 \\
23 \\
25 \\
22 \\
35 \\
25 \\
15 \\
27 \\
33 \\
\end{array}$ & & $\begin{array}{l}85 \\
54 \\
60 \\
69 \\
65 \\
61 \\
55 \\
54 \\
49 \\
\end{array}$ & 9 & $\begin{array}{l}1 \\
1\end{array}$ & $\begin{array}{l}1 \\
1 \\
\end{array}$ & $\begin{array}{l}1 \\
1 \\
1 \\
1 \\
1\end{array}$ & $\begin{array}{r}23 \\
15 \\
\\
14 \\
10 \\
11 \\
8 \\
\end{array}$ & $\begin{array}{r}20 \\
8 \\
10 \\
\end{array}$ & \\
\hline \multicolumn{19}{|l|}{ Hole 401} \\
\hline Quaternary & 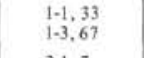 & $\begin{array}{l}0.35 \\
3.67\end{array}$ & $\begin{array}{l}20 \\
26\end{array}$ & $\begin{array}{r}32 \\
9\end{array}$ & & & & $1 \mid \begin{array}{l}10 \\
10\end{array}$ & $\begin{array}{l}0 \\
0 \\
0\end{array}$ & $\begin{array}{l}30 \\
20\end{array}$ & & $\begin{array}{l}20 \\
30\end{array}$ & & & 1 & & & \\
\hline Early Otigocene & $2-1.7$ & 84.57 & 3 & 71 & & & & & & & & & & & & & & \\
\hline Late Eocene & $\begin{array}{l}3-1,15 \\
4, \mathrm{CC}\end{array}$ & $\begin{array}{r}94.15 \\
103.50\end{array}$ & $\begin{array}{l}7 \\
4\end{array}$ & $\begin{array}{l}59 \\
68\end{array}$ & & & & & $\begin{array}{l}20 \\
20\end{array}$ & & $\begin{array}{l}60 \\
65\end{array}$ & $\begin{array}{l}15 \\
10\end{array}$ & & & 1 & 5 & & \\
\hline Middle Eocene & 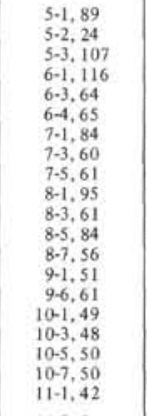 & $\begin{array}{l}113.89 \\
114.74 \\
117.07 \\
123.66 \\
126.14 \\
127.65 \\
132.84 \\
135.60 \\
138.61 \\
142.45 \\
145.11 \\
148.34 \\
151.06 \\
151.51 \\
159.11 \\
160.99 \\
163.98 \\
167.00 \\
170.00 \\
170.42\end{array}$ & $\begin{array}{l}6 \\
5 \\
9 \\
7 \\
8 \\
8 \\
5 \\
6 \\
6 \\
9 \\
9 \\
7 \\
7 \\
9 \\
8 \\
7 \\
8 \\
5 \\
9 \\
8\end{array}$ & \begin{tabular}{|l|}
60 \\
55 \\
37 \\
46 \\
45 \\
49 \\
57 \\
45 \\
40 \\
42 \\
41 \\
45 \\
38 \\
41 \\
49 \\
46 \\
44 \\
51 \\
54 \\
54
\end{tabular} & & 1 & & & $\begin{array}{l}20 \\
20 \\
15 \\
30 \\
25 \\
15 \\
30 \\
20 \\
40 \\
30 \\
30 \\
30 \\
25 \\
20 \\
25 \\
20 \\
25 \\
25 \\
10\end{array}$ & 15 & $\begin{array}{l}80 \\
80 \\
85 \\
70 \\
75 \\
70 \\
70 \\
80 \\
60 \\
70 \\
70 \\
70 \\
75 \\
75 \\
75 \\
75 \\
70 \\
70 \\
85\end{array}$ & & $\begin{array}{l}1 \\
1 \\
1\end{array}$ & $\begin{array}{l}1 \\
1\end{array}$ & $\begin{array}{l}1 \\
1 \\
1 \\
1 \\
1 \\
1 \\
1 \\
1\end{array}$ & $\mid \begin{array}{l}5 \\
5 \\
5 \\
5 \\
5 \\
\end{array}$ & & \\
\hline Early Eocene & $\begin{array}{l}11-3,9 \\
11-3,91 \\
11-5,70 \\
12-1,49 \\
12-3,111 \\
12-4,28\end{array}$ & $\begin{array}{l}173.09 \\
173.91 \\
176.70 \\
179.99 \\
183.61 \\
184.28 \\
\end{array}$ & $\begin{array}{l}4 \\
6 \\
4 \\
5 \\
4 \\
2 \\
\end{array}$ & $\begin{array}{l}57 \\
33 \\
46 \\
50 \\
44 \\
72\end{array}$ & & 1 & & $\begin{array}{l}15 \\
20 \\
30 \\
10\end{array}$ & $\begin{array}{l}10 \\
15 \\
10 \\
15 \\
15 \\
15\end{array}$ & & \begin{tabular}{|l|}
80 \\
80 \\
60 \\
55 \\
55 \\
50 \\
\end{tabular} & $\begin{array}{l}10 \\
10 \\
10 \\
\end{array}$ & & $\begin{array}{l}1 \\
1 \\
1\end{array}$ & 1 & \begin{tabular}{r|}
10 \\
5 \\
5 \\
15 \\
15
\end{tabular} & & \\
\hline
\end{tabular}


TABLE 2 - Continued

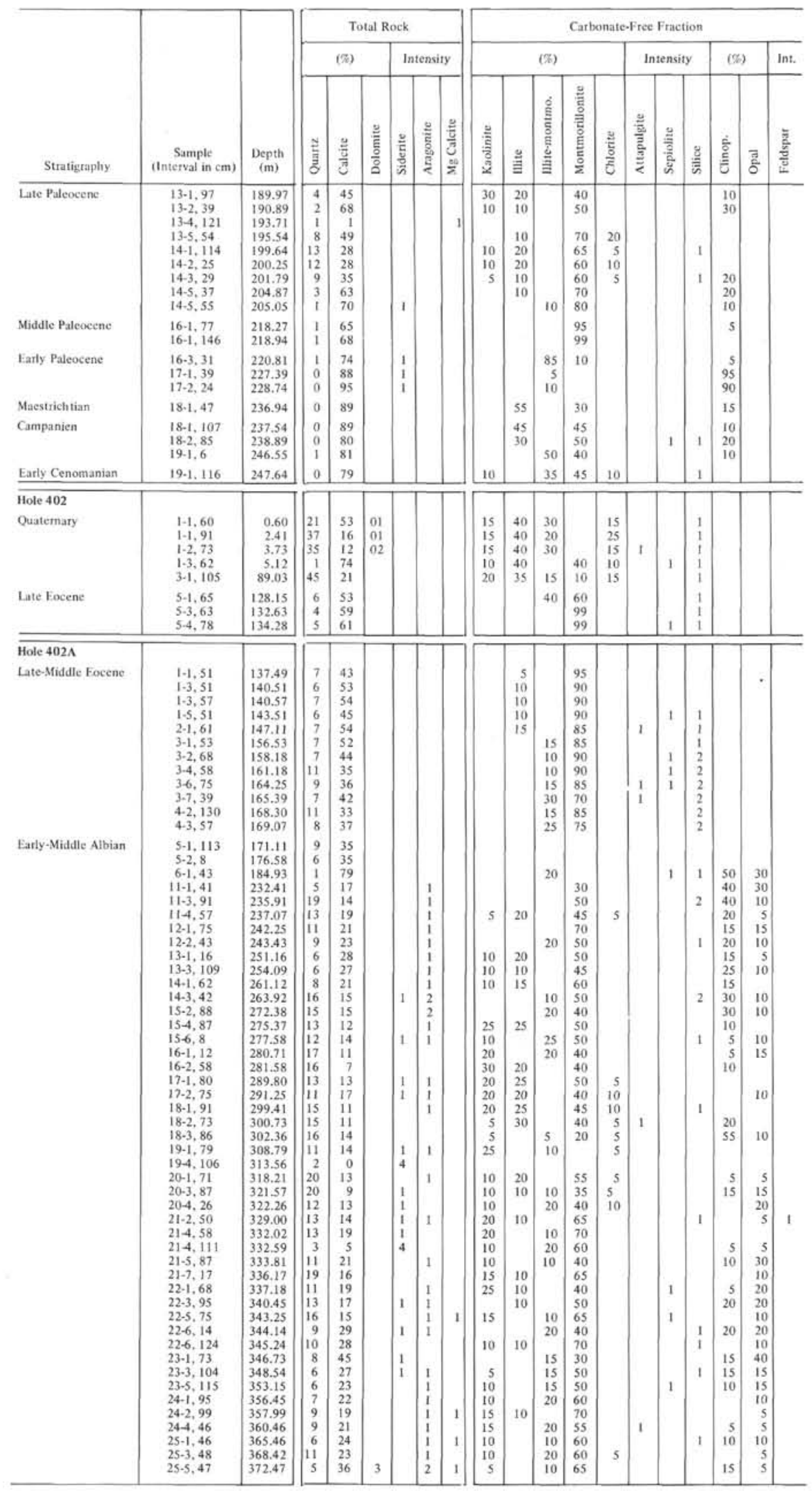


TABLE 2 - Continued

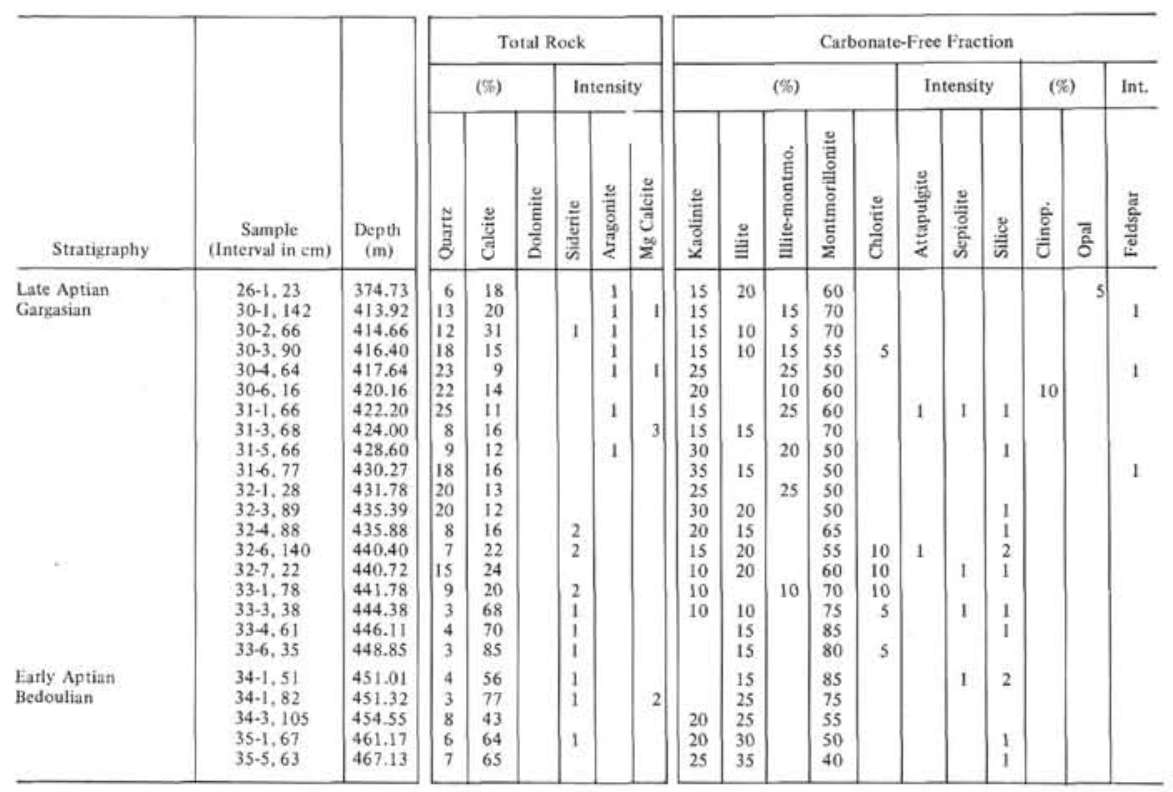





\section{PLATE 1}

Carbonate-free fine-fraction sediments, stereoscan photographs and X-ray diagrams.

Figure $1 \quad$ Opal-CT and zeolite crystallization from Sample 400A-63, CC.

Figure $2 \quad \mathrm{X}$-ray diagram corresponding to Figure 1.

Figure 3 Bioclastics remains from Sample 401-16-1, $146 \mathrm{~cm}$.

Figure $4 \quad \mathrm{X}$-ray diagram corresponding to Figure 3.

Figure 5 Clinoptilolite crystallization from Sample 401-17-1, $39 \mathrm{~cm}$.

Figure $6 \quad \mathrm{X}$-ray diagram corresponding to Figure 5. 
PLATE 1
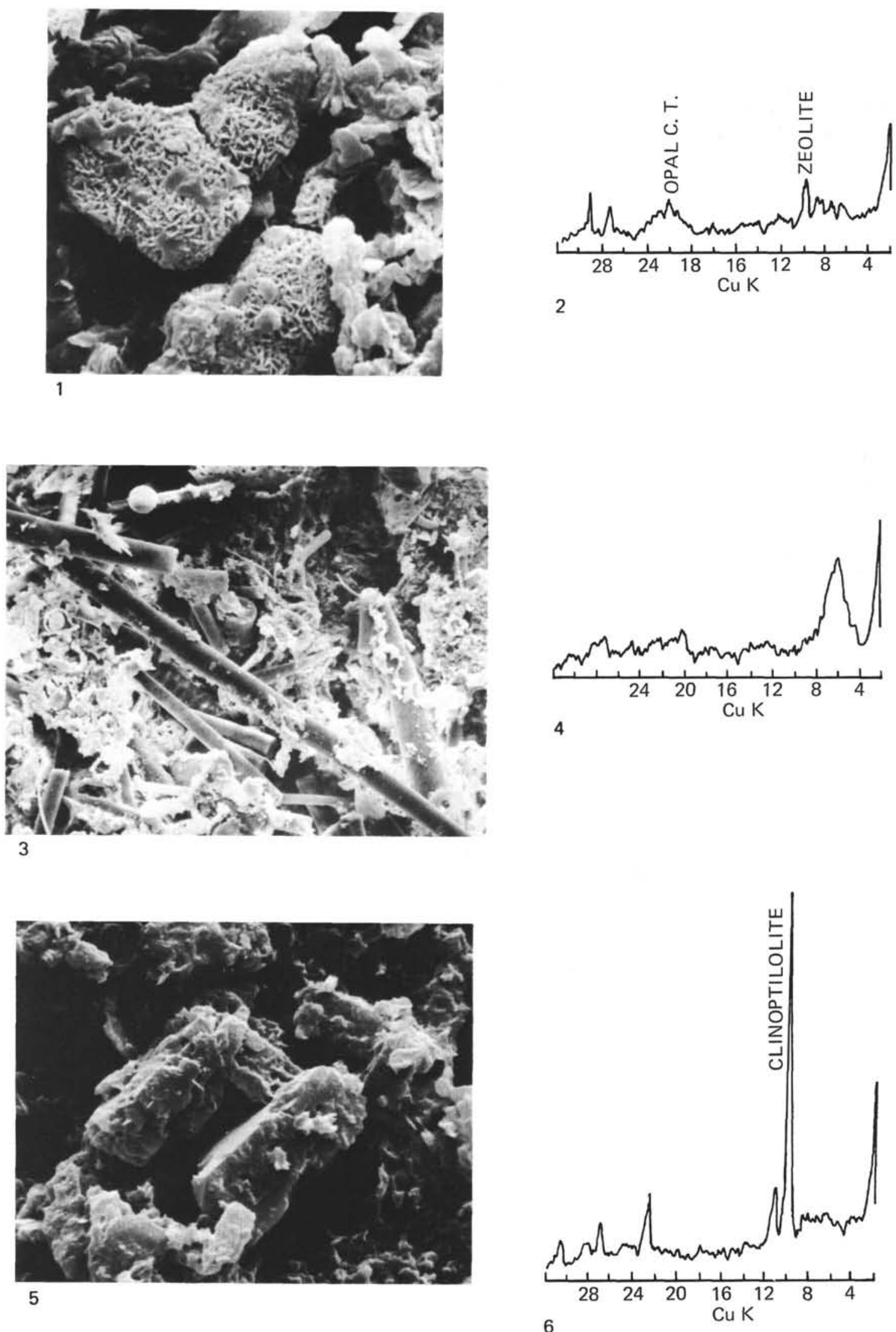


\section{PLATE 2}

Carbonate-free fine-fraction sediments, stereoscan photographs and $\mathrm{X}$-ray diagrams.

Figure 1 Detail of zeolite crystallization from Sample $401-17-1,39 \mathrm{~cm}$.

Figure 2 Clinoptilolite crystallization from Sample 401-17-2, $24 \mathrm{~cm}$.

Figure 3 Opal-CT crystallization from Sample 402A-29-3, $104 \mathrm{~cm}$.

Figure $4 \quad$ Detail of Figure 3.

Figure $5 \quad \mathrm{X}$-ray diagram corresponding to Figure 2.

Figure $6 \quad \mathrm{X}$-ray diagram corresponding to Figure 3. 


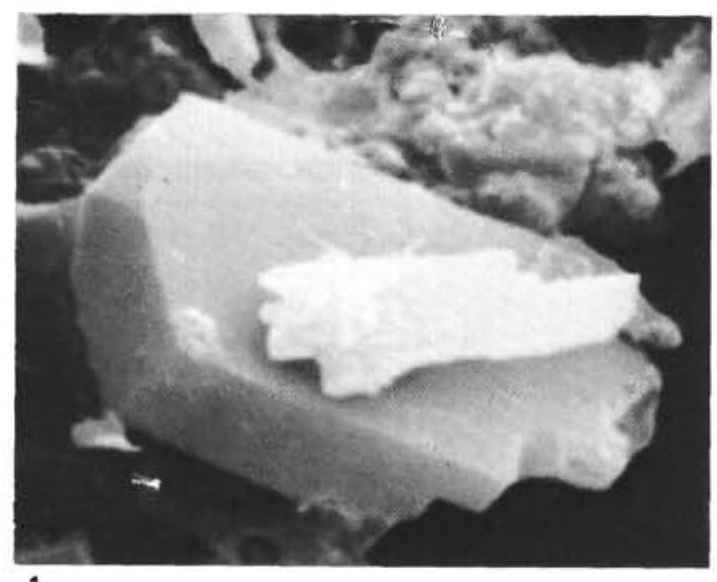

1
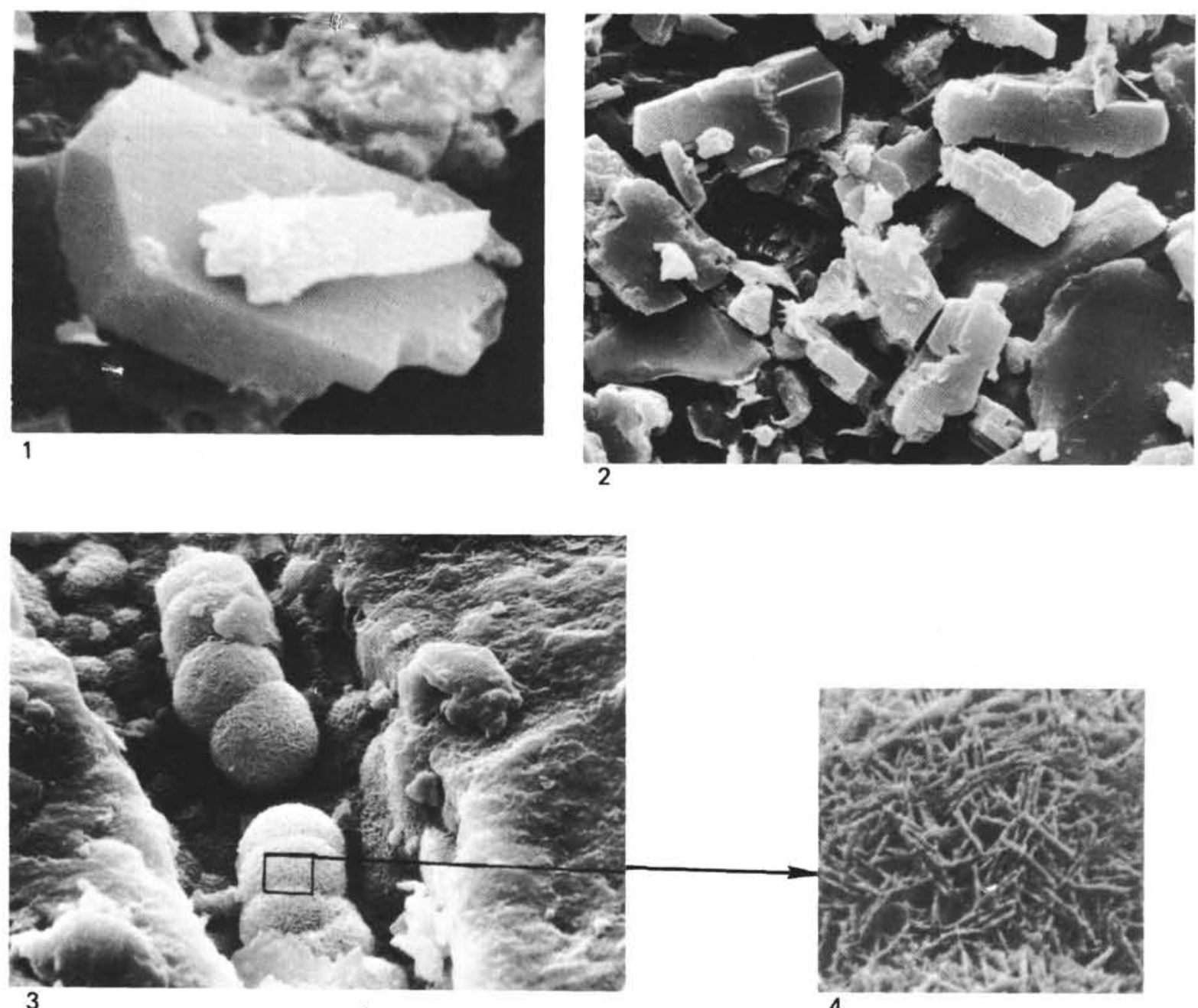

3
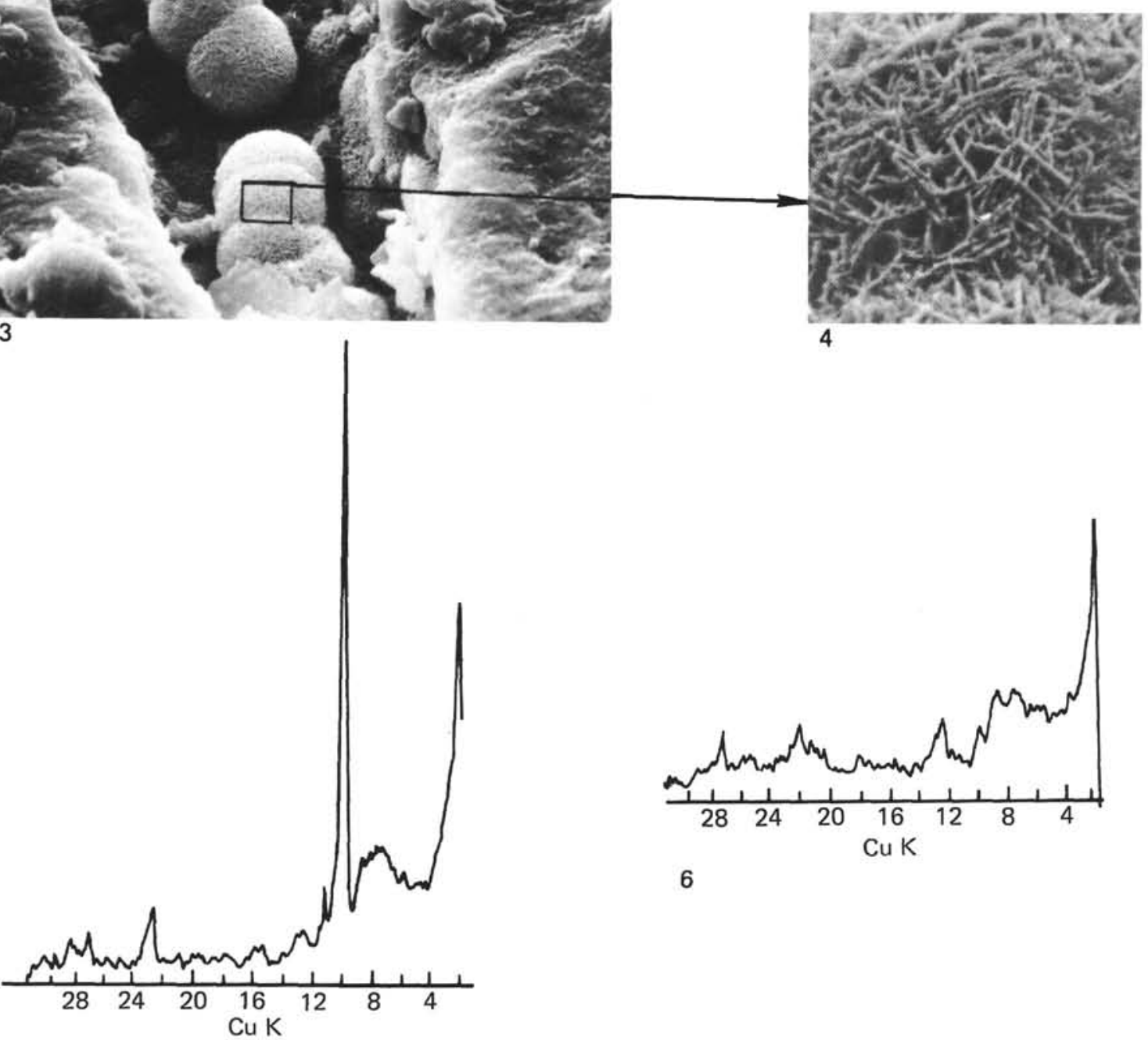

6

5 